\title{
Estimating the Risk of Chemotherapy Toxicity in Indian Geriatric Patient Population and Utility of Chemotherapy Risk Assessment Scale for High Age Patients (CRASH) Score
}

\author{
Aditi Mittal ${ }^{1}$ Ranga R. Rangaraju ${ }^{2} \quad$ Amit Agarwal $^{3} \quad$ Chandragouda D. $^{3} \quad$ Sandeep Batra $^{4}$ Suhail Qureshi
}

${ }^{1}$ Department of Medical Oncology, Sawai Man Singh Medical College, Jaipur, Rajasthan, India

2Department of Medical Oncology, Max Super Speciality Hospital, Shalimar Bagh, New Delhi, India

${ }^{3}$ Department of Medical Oncology, B.L. Kapur Super Speciality Hospital, Rajendra Place, New Delhi, India

${ }^{4}$ Department of Medical Oncology, Max Super Speciality Hospital, Saket, Delhi, India

\section{South Asian J Cancer 2021;10:161-166.}

Address for correspondence Aditi Mittal, MBBS, MD (Medicine), DrNB (Medical oncology) Department of Medical Oncology, Sawai Man Singh Medical College , Jaipur 302004, Rajasthan, India (e-mail: aditi120985@gmail.com).
Abstract

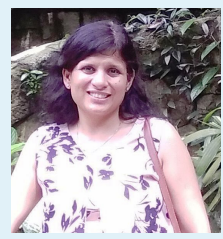

Aditi Mittal
Background Aging is a heterogeneous process, and elderly population is diverse in health status and functional reserve. The present study was undertaken to predict severe chemotherapy toxicity using the Chemotherapy Risk Assessment Scale for High-Age Patients' (CRASH) score.

Materials and Methods Elderly patients (age $\geq 65$ years) with malignancy, who were planned to be treated with a new course of cytotoxic chemotherapy, were enrolled. The CRASH score was calculated, and patients were stratified into four categories, that is, low (0-3), intermediate (Int)-low (4-6), Int-high (7-9), and high (<9). Patients developing grade $3 / 4 / 5$ nonhematologic $(\mathrm{NH})$ or grade $4 / 5$ hematologic $(\mathrm{H})$ toxicity were taken as the development of severe toxicity.

Results Of 100 enrolled patients, 64 (64\%) were able to complete their prescribed treatment. Forty-four percent of patients (44 patients) of our study cohort experienced grade- $4 \mathrm{H}$ or grade $3 / 4 \mathrm{NH}$ toxicity. The highest score in each category (heme/nonheme/CRASH) predicts nearly $100 \%$ toxicity risk. At a critical value of CRASH $\geq 6.5$, the sensitivity is calculated as $100 \%$, while specificity is $89.09 \%$. The accuracy of prediction is $93.88 \%$. The median time taken to develop toxicity was 39.5 days.

Keywords

- chemotherapy

- elderly

- risk assessment

- toxicity
Conclusion CRASH score utilizes clinical assessment and basic laboratory values. Yet, it accurately predicts severe chemotherapy toxicity above a critical value of 6.5. Based on the above study, the first 30 days are crucial as $45 \%$ of patients experienced toxicity in this time frame. With the help of these clinical predictive markers, the care of elderly will be optimized.
How to cite this article: Mittal A, Rangaraju R. R, Agarawal A, et al. Estimating the Risk of Chemotherapy Toxicity in Indian Geriatric Patient Population and Utility of Chemotherapy Risk Assessment Scale for High-Age Patients' Score South Asian J Cancer 2021; 10(3):161-166.
(C) 2021. MedIntel Services Pvt Ltd.

This is an open access article published by Thieme under the terms of the Creative Commons Attribution-NonDerivative-NonCommercial-License, permitting copying and reproduction so long as the original work is given appropriate credit. Contents may not be used for commercial purposes, or adapted, remixed, transformed or built upon. (https://creativecommons.org/licenses/by-nc-nd/4.0/).

Thieme Medical and Scientific Publishers Private Ltd A-12, Second Floor, Sector -2, NOIDA -201301, India 


\section{Introduction}

The field of geriatrics is gradually being recognized as the need of the century. Throughout the world, all populations are seeing the burgeoning numbers of elderly. As per the latest census of 2011 in India, the population aged above 65 years comprises $5 \%$ ( $4.8 \%$ urban and $5.1 \%$ rural $).{ }^{1}$ Cancer is a disease of aging, with the majority falling in the age group above 65 years. $^{2}$

Data from earlier trials and meta-analysis provide conflicting results regarding the benefit of chemotherapy in elderly ${ }^{3-5}$ An Indian study ${ }^{6}$ noted an increased dropout rate or discontinuation of treatment in elderly compared with younger population.

As a standard oncology evaluation cannot recognize those with the likelihood of toxicity due to treatment, objective and measurable factors are needed for rational decision-making. Few predictive scores are available to assess the individual risk of severe toxicity, namely, the Cancer and Aging Research Group (CARG), ${ }^{7}$ Chemotherapy Risk Assessment Scale for High-Age Patients' (CRASH) score, ${ }^{8}$ and Get up and Go test. ${ }^{9}$ CRASH is a more comprehensive, detailed, and informative score. CRASH utilizes the MAX 2 inde ${ }^{10}$ for estimating the chemotherapy toxicity. The MAX2 index is a convenient and reproducible way of comparing the average per patient risk for toxicity from chemotherapy across several regimens.

Hence, this exploratory study was undertaken if we can predict severe chemotherapy toxicity using the CRASH score as a model at a tertiary health care center. It is perhaps the first study quoting predictive factors for chemotherapy toxicity in the Indian geriatric population.

\section{Materials and Methods}

The present study was a single-institution prospective observational cohort study conducted in the Department of Medical Oncology at Dr. B.L. Kapur Hospital, Pusa Road, New Delhi, from October 2014 to 2016. Patients were enrolled as per the type-1 progressive censoring scheme. As per the institution load, it was decided to conduct a pilot study, and 100 consecutive patients were enrolled as per the following inclusion and exclusion criteria. Patients with (1) age $\geq 65$ years, (2) histologic documentation of malignancy, (3) planned for new course of cytotoxic chemotherapy, (4) able to answer questions, (5) willing to give consent, (6) received radiation $>3$ months before or after the completion of chemotherapy were included in the study. Patients (1) undergoing radiation therapy < 3 months, (2) have complaints of dementia and are in altered behavior or sensorium, (3) undergoing chemotherapy for bone marrow transplant, and (4) unwilling to give consent were excluded from the study.

All patients were evaluated with a detailed history and physical examination. The staging was performed as per routine clinical practice. Standard chemotherapy was planned by the treating physician. Biochemical, hematological, and radiological tests were done before recruitment and on follow-up.
The Eastern Cooperative Oncology Group (ECOG) performance status (PS), Lawton nine-item Instrumental Activities of Daily Living (IADL), Mini Nutritional Assessment (MNA), and Folstein Mini-Mental Status Examination (MMSE) were administered by principal investigator verbally, and results were recorded. The risk of severe chemotherapy toxicity was computed using the MAX2 index..$^{10}$ Briefly, the MAX2 index is average of the highest frequency of both grade- 4 hematologic $(\mathrm{H})$ and grade $3 / 4$ nonhematologic $(\mathrm{NH})$ toxicity. It is reproducible across cancer types and is sensitive to toxicity differences. The component of CRASH score ${ }^{8}$ and its variables are shown in - Table 1. The CRASH score varies from 0 to 12 . Patients were stratified into four categories, that is, low $(0-3)$, intermediate (Int)-low (4-6), Int-high (7-9), and high (<9). All procedures followed were in accordance with the ethical standard of the responsible committee on human experiments (institutional and national) and with the 1975 Declaration of Helsinki, as revised in 2008. ${ }^{5}$ Written informed consent was obtained from all patients before the enrollment.

Toxicity was graded as per CTCAE (Common Terminology Criteria for Adverse Events) adverse events criteria version 4.0, published on May 28, 2009. Patients developing grade $3 / 4 / 5 \mathrm{NH}$ or grade $4 / 5 \mathrm{H}$ toxicity were taken as the development of severe toxicity.

\section{Statistical Analysis}

The quantitative variables were expressed as mean \pm standard deviation (SD) and compared using unpaired $t$-test. Data grouped in contingency tables, wherein the Chi-square test was used to assess the associations. Receiver operating characteristic (ROC) curves were made to identify the critical values, and, hence, odds ratio, sensitivity, and specificity were calculated. Kaplan-Meier product limit estimator is used to calculate the expected median survival time. A $p$-value of $<0.05$ is considered as statistically significant. (SPSS, International Business Machines Corporation, Armonk, New York, United States) version 16.0 software is used for statistical analysis.

Table 1 Components of the chemotherapy risk assessment scale for high-age patients score

\begin{tabular}{|l|l|}
\hline $\begin{array}{l}\text { Chemotherapy risk (chemotox } \\
\text { score) as per MAX2 index }\end{array}$ & $0-0.44$ (score 0$)$ \\
\cline { 2 - 2 } & $0.45-0.57$ (score 1$)$ \\
\cline { 2 - 2 } $\begin{array}{l}\text { Hematologic risk factors and } \\
\text { scoring }\end{array}$ & $\begin{array}{l}\text { Diastolic blood pressure } \\
(>72 \mathrm{~mm} \mathrm{Hg}=1)\end{array}$ \\
\cline { 2 - 2 } & $\mathrm{IADL}(<26=1)$ \\
\cline { 2 - 2 } & $\mathrm{LDH}(>459=2)$ \\
\hline \multirow{2}{*}{$\begin{array}{l}\text { Nonhematologic risk factors and } \\
\text { scoring }\end{array}$} & $\mathrm{ECOG}$ PS $(1-2=1 ; 3-4=2)$ \\
\cline { 2 - 2 } & MMSE $(<30=2)$ \\
\cline { 2 - 2 } & MNA $(<28=2)$ \\
\hline
\end{tabular}

Abbreviations: ECOG, Eastern Cooperative Oncology Group; IADL, instrumental activities of daily living; LDH, lactate dehydrogenase; MMSE, Mini-Mental Status Examination; MNA, Mini Nutritional assessment; PS, performance status. 


\section{Results}

Patient, disease, and treatment characteristics are shown in - Table 2. Most of the patients had one or more comorbidity (61\%), the most common being diabetes mellitus (41\%). Chemotherapy-related toxicity was $51.2,27.5$, and $50 \%$ in patients with zero, one, more than one comorbidity ( $p=$ 0.107 ). No association could be deduced between chemotherapy toxicity and carcinoma site due to a small sample size and varied histopathology. In total, 29 different chemotherapy regimens and schedules were used. Weekly paclitaxel and carboplatin were most commonly used regimens (26\%) followed by nab-paclitaxel (11\%). Among patients with metastatic setting, $67 \%$ of them received first-line chemotherapy. A subset of patients (8) was receiving third-line chemotherapy and they experienced maximum toxicity (six out of eight patients) $(p=0.234)$. Although $p$-value was not statistically significant, still this points toward the role of cumulative chemotherapy toxicity.

Geriatric assessment variables were a critical part of the predictive model. Three-fourth of the patients had ECOG PS $\leq 2$ (76 out of 100 ), as shown in - Table 2 . More than $90 \%$ of patients with PS-3 developed toxicity in comparison to $23.3 \%$ in patients with PS-1 $(p<0.05)$. The mean score on IADL was 23.03 ( $S D=4.02$, range: $13-29$ ), with $68 \%$ of them having a score $<26$, and functionally disabled in one or more IADL. The mean MMSE score was 28.47 ( $S D=2.72$, range: $16-30$ ); 49 with normal cognition. The mean score of MNA was 20.7 (range: 12-28.5). Twenty percent of the patient population was found to be severely malnourished (MNA score <17), and $53 \%$ was at risk. The incidence of toxicity was the highest in the malnourished group (55.5\%). Body mass index alone was not a good tool for evaluating nutritional status in an individual $(p=0.61)$.

Amidst the various laboratory variables, mean hemoglobin, albumin, and serum lactic dehydrogenase values were $11.3,3.65$, and $322 \mathrm{~g} / \mathrm{dL}$, respectively. The value of serum lactate dehydrogenase (LDH) was greater than twice the normal in $13 \%$ of patients (range: 106-3,224).

Overall, 64 (64\%) patients were able to complete their prescribed treatment. Twelve patients stopped or changed to another chemotherapy regimen due to disease progression. Twenty-four patients (24\%) stopped the treatment due to toxicity. Among patients who stopped treatment , most of them had advanced disease (22 out of 24 ) and poor PS. More than half of them (54\%)received polychemotherapy. Two patients were lost to follow-up and their data were censored till the last follow-up.

Forty-four percent of patients (44 patients) of our study cohort experienced grade- $4 \mathrm{H}$ or grade $3 / 4 \mathrm{NH}$ toxicity, $13 \%$ (13) had grade- $4 \mathrm{H}$ toxicity, and $42 \%$ (42 patients) had grade $3 / 4 \mathrm{NH}$ toxicity. Three patients (3\%) died within 1 month of starting treatment. Maximum events (45.4\%) occurred in the first month of starting chemotherapy.

-Fig. 1 shows the four groups as per the CRASH score and the occurrence of chemotherapy-related toxicity. The highest score in each category (heme/nonheme/CRASH) predicts nearly $100 \%$ toxicity risk. The association between CRASH score and toxicity (Chi-square $p<0.001$ ) is found to
Table 2 Baseline demographic and clinical characteristics of elderly patients

\begin{tabular}{|c|c|}
\hline Variable & $n$ \\
\hline Age (y) & $65-80$ \\
\hline Mean \pm SD & $68.46 \pm 4.3$ \\
\hline 65-74 (young old) & 90 \\
\hline $75-84$ (old-old) & 10 \\
\hline >85 (oldest old) & 0 \\
\hline \multicolumn{2}{|l|}{ Sex } \\
\hline Female & 44 \\
\hline Male & 56 \\
\hline \multicolumn{2}{|l|}{ Comorbidities } \\
\hline $0-1$ & 68 \\
\hline$>1$ & 32 \\
\hline \multicolumn{2}{|l|}{ Tumor site } \\
\hline Gl & 27 \\
\hline Carcinoma ovary (including PPC) & 21 \\
\hline Breast carcinoma & 14 \\
\hline Carcinoma lung & 13 \\
\hline NHL (DLBCL) & 8 \\
\hline Genitourinary cancer & 8 \\
\hline Head and neck cancer & 7 \\
\hline Synovial sarcoma & 2 \\
\hline \multicolumn{2}{|l|}{ Disease extent, stage wise } \\
\hline 1 & 3 \\
\hline 2 & 11 \\
\hline 3 & 17 \\
\hline 4 & 69 \\
\hline \multicolumn{2}{|l|}{ Intent of chemotherapy } \\
\hline $\begin{array}{l}\text { Definitive (adjuvant/curative/ } \\
\text { neoadjuvant) }\end{array}$ & 35 \\
\hline Palliative & 65 \\
\hline \multicolumn{2}{|l|}{ ECOG PS } \\
\hline $0-1$ & 32 \\
\hline$>1$ & 68 \\
\hline \multicolumn{2}{|l|}{ Serum lactic dehydrogenase (IU/L) } \\
\hline Score $2(>459)$ & 13 \\
\hline Score $0(<459)$ & 87 \\
\hline \multicolumn{2}{|l|}{ Chemotherapy } \\
\hline Monochemotherapy & 31 \\
\hline Polychemotherapy (>1 drug) & 69 \\
\hline \multicolumn{2}{|l|}{ Chemotherapy regimens (most common) } \\
\hline Weekly paclitaxel + carboplatin & 26 \\
\hline Weekly nab-paclitaxel & 11 \\
\hline FOLFOX/CAPOX & 10 \\
\hline \multicolumn{2}{|l|}{ Use of G-CSF } \\
\hline Yes & 77 \\
\hline No & 23 \\
\hline
\end{tabular}

Abbreviations: DLBCL, diffuse large B cell lymphoma; ECOG, Eastern Cooperative Oncology Group; FOLFOX (folinic acid, fluorouracil, and oxaliplatin) CAPOX (capecitabine, oxaliplatin); G-CSF, growth colony-stimulating factor; $\mathrm{Gl}$, gastrointestinal; NHL, non-Hodgkin's lymphoma; PPC, primary peritoneal cancer; PS, performance status; SD, standard deviation. 
be statistically significant. The mean CRASH score among patients who developed toxicity was $8.52 \pm 1.19$ versus 5.15 $\pm 1.33(p<0.001)$. Similar observations were made for heme and nonheme scores.

The ROC of the CRASH model with chemotherapy toxicity produces area under the curve as $97 \%(p<0.001)$ which has a high statistical significance value. Choosing a critical value of CRASH score of $\geq 6.5$ for predicting toxicity, the sensitivity is calculated as $100 \%$, while specificity is $89.09 \%$. The accuracy of prediction is $93.88 \%$. The area under the ROC curve is $94.3 \%(p<0.001)$ and $90.7 \%$ for heme and nonheme score, respectively. The accuracy of prediction is around $90 \%$ for both models.

The mean survival time was 540 days for patients who did not develop toxicity versus 264 days for those who developed toxicity. The difference between the groups is statistically

Figure 1:Association of CRASH score and toxicity

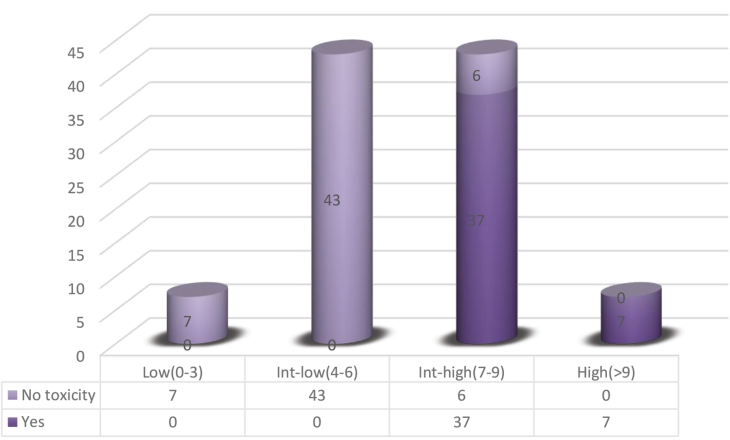

Fig. 1 Association of the Chemotherapy Risk Assessment Scale for High-Age Patients' (CRASH) score and severe chemotherapy toxicity. This figure shows that patients with the low Chemotherapy Risk Assessment Scale for High-Age Patients score are at $0 \%$ risk of toxicity versus patients at other end with high score have $100 \%$ toxicity. Int, intermediate.

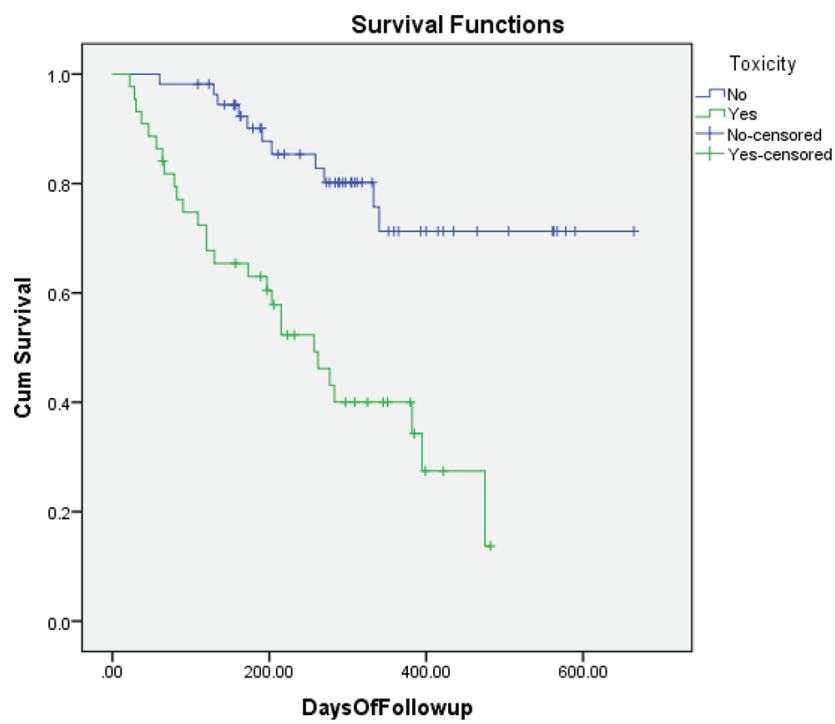

Fig. 2 Kaplan-Meier curves of those who developed toxicity versus who do not. Kaplan-Meier curves of patients those who developed toxicity versus who do not. The figure depicts the rapidly falling curve for the patients who develop toxicity and hence decreased median survival. significant $(p<0.005)$. The Kaplan-Meier curves are shown in -Fig. 2. Overall, $37 \%$ of our study patients died during the study.

\section{Discussion}

This prospective, observational, single hospital-based study evaluated risk factors for the prediction of chemotherapy toxicity in elderly population ( $\geq 65$ years) with the usage of the CRASH score. In this study, CRASH was found to be a good predictive tool above a value of 6.5 to anticipate chemotherapy-related severe toxicity - Table 3 (accuracy 93.88\%). More than half (64\%) of patients completed treatment, and $44 \%$ experienced severe chemotherapy-related toxicity.

Approximately 11 lakh new cancer patients are being diagnosed in India yearly (GLOBOCAN 2018). ${ }^{11}$ About 12 to 23\% of all cancer occurs in elderly ( $\geq 65$ years). ${ }^{12}$ Thus, in years to come, treating physician/oncologist will face a huge number of older patients with cancer. Chemotherapy works not only for improving the quantity of life but also to improve the quality of life. Effective management of chemotherapy-related toxicity with appropriate supportive care is crucial. The International Society of Geriatric Oncology and National Comprehensive Cancer Network advise performing some form of geriatric assessments in all older patients with cancer. ${ }^{13}$ As suggested by the previous studies, geriatric assessment can lead to modification in treatment planning in 20 to $50 \%$ of patients. ${ }^{14} \mathrm{CRASH}^{8}$ is one such tool with high sensitivity and specificity. It utilizes various patients, diseases, and chemotherapy-related variables and creates a comprehensive landscape for the clinician. Among others, the CARG model $^{7}$ distributes patients into three groups with the occurrence of toxicity as $36.7,62.4$, and $70.2 \%$ in low-, medium-, and high-risk groups, respectively $(p<0.001)$. The notable difference between these models is that CRASH is exhaustive, and in addition, it defines $\mathrm{H}$ and $\mathrm{NH}$ toxicities separately.

Chemotherapy-related toxicities in elderly vary across studies ranging from 28 to $64 \% .^{7,8,15,16}$ Sparse data are available from India concerning this problem. Sarkar and Shahi ${ }^{6}$ reported treatment (radiation, surgery, and chemotherapy)-related grade $3 / 4$ toxicity in elderly as $10.2 \%$ (4/39 patients). We sought to identify lone chemotherapy-related toxicity in this cohort. $\mathrm{H}$ and $\mathrm{NH}$ toxicities were experienced by 13 and 42 patients, respectively. Older adults seem to be susceptible to increased myelosuppression due to limited hematopoietic reserve. ${ }^{17}$ Balducci and Corcoran ${ }^{17}$ mentioned that myelosuppression can be reduced with the use of growth factors. We observed limited $\mathrm{H}$ toxicity (13\%) due to the liberal use of growth factors (77\%) and the exclusion of leukemia and high-dose therapy ( - Table 3 ).

Patients with PS-3 developed toxicity in 91\% patients in comparison with $23.3 \%$ in PS-1 $(p<0.05)$. In addition, patients with stage- 4 disease has higher toxicity ( 49.2 vs. $32.2 \%$ ) but statistically insignificant. Similarly, Freyer et $\mathrm{al}^{15}$ deduced depression ( $p=0.006)$, PS $\geq 2(p=0.026)$, and dependence ( $p$ $=0.048$ ); FIOGO (The International Federation of Gynecology 
Table 3 Statistical parameters of the Chemotherapy Risk Assessment Scale for High-Age Patients' score

\begin{tabular}{|c|c|c|c|c|c|c|c|c|}
\hline Toxicity CRASH score & Yes $n(\%)$ & No $n(\%)$ & $p$ & Sensitivity (\%) & Specificity (\%) & PPV (\%) & NPV (\%) & Accuracy (\%) \\
\hline$<6.5$ & $0(0.00)$ & $50(89.28)$ & \multirow[t]{2}{*}{$<0.001$} & \multirow[t]{2}{*}{100.00} & \multirow[t]{2}{*}{89.09} & \multirow[t]{2}{*}{87.76} & \multirow[t]{2}{*}{100.00} & \multirow[t]{2}{*}{93.88} \\
\hline$\geq 6.5$ & $44(100.00)$ & $6(10.71)$ & & & & & & \\
\hline Total & $44(100)$ & $56(100)$ & & & & & & \\
\hline
\end{tabular}

Abbreviations: CRASH, Chemotherapy Risk Assessment Scale for High-Age Patients' score; NPV, negative predictive value; PPV, positive predictive value.

and Obstetrics) stage IV $(p=0.075)$ as risk factors for developing toxicity.

The components of the CRASH score are equally useful. The odds of developing $\mathrm{H}$ toxicity were found to be 125 times more when heme score $>3.5$. This information can be utilized to categorize patients in whom either dose modification or the use of growth factors is warranted. Akin to this, $\mathrm{NH}$ score of $>5.5$ entails an individual 40 times prone for adverse events.

Crawford et $\mathrm{al}^{18}$ and Lyman et $\mathrm{al}^{19}$ stated the maximum occurrence of $\mathrm{H}$ toxicity after the first cycle of chemotherapy. In our cohort, the maximum number of events (45.4\%) occurred in first month of starting chemotherapy. To infer, one should carry close and frequent monitoring during this period.

Authors have employed 10 to 45 minutes to conduct abbreviated comprehensive geriatric assessment (aCGA), ${ }^{8,20,21}$ In a resource-strained country like India, it is a daunting process to allot around one and half to conduct this score. The feasibility in day-to-day practice is matter of concern.

The aCGA predicts mortality as shown by Fried's operational criteria for frailty, ${ }^{22}$ functional status, ${ }^{23}$ cognitive impairment, ${ }^{24}$ nutrition, ${ }^{25}$ and depression. ${ }^{25}$ On the other hand, Puts et $\mathrm{al}^{21}$ failed to find a correlation between frailty markers and mortality. The mean time of survival was significantly worse for those who developed toxicity $(p<0.005)$. The CRASH score was associated with the development of toxicity, and latter is a risk factor for decreased survival. Consequently, CRASH may also serve as an indirect measure of survival.

\section{Limitations}

The limitations of the study are as follows: the study was conducted in a single center with small $(n=100)$ and heterogeneous cancer population. We reported only grades 3 to 5 toxicity, although grade- 2 toxicities (diarrhea and neuropathy) may also be pertinent to the geriatric population. The aCGA was done once; however, longitudinal evaluation would be more informative. CRASH is still not validated for targeted, oral, and immunotherapy. However, our results show that some simple parameters can be systematically assessed to guide the physicians to choose the best therapeutic strategy.

\section{Conclusion}

We reported the incidence of lone chemotherapy-related toxicity, perhaps for the first time in the Indian context.
Although the CRASH score is time consuming and exhaustive, it correlated well with anticipation of toxicity. For frail elderly, the first 30 days are most crucial. The mean survival and number of chemotherapy cycles received are adversely affected with the development of toxicity. Large-scale disease-specific studies are needed to identify clinical and laboratory factors affecting the development of toxicity.

Funding

Conflict of Interest

There are no conflicts of interest to declare.

\section{Acknowledgments}

I am extremely grateful to all administrative and clinical staff at Dr. B.L. Kapur Memorial Hospital, Pusa Road, New Delhi, for granting me permission to carry out this study. I am deeply indebted to all my patients and their families who agreed to be a part of this study. I pay my respect to those (my patients) who are no longer in this world.

\section{References}

1 Statista, Hamburg, Germany. Available at https://www. statista.com/statistics/271315/age-distribution-in-india/, Accessed April 12, 2021. Accessed August 17, 2013

2 Howlader N, Noone AM, Krapcho M, Miller D, Bishop K, Altekruse SF, Kosary CL, Yu M, Ruhl J, Tatalovich Z, Mariotto A, Lewis DR, Chen HS, Feuer EJ, Cronin KA (eds). SEER Cancer Statistics Review, 1975-2013, National Cancer Institute. Bethesda, MD, https://seer.cancer.gov/archive/csr/1975_2013/, based on November 2015 SEER data submission, posted to the SEER web site, April 2016. Accessed on April 12,2021

3 Clarke M, Collins R, Darby S, et al. Early breast cancer trialists' collaborative group (EBCTCG). Effects of chemotherapy and hormonal therapy for early breast cancer on recurrence and 15-year survival: an overview of the randomized trials. Lancet 2005;366:2087-2106

4 Pignon JP, Bourhis J, Domenge C, Designé L. Chemotherapy added to locoregional treatment for head and neck squamous-cell carcinoma: three meta-analyses of updated individual data. MACH-NC Collaborative Group. Meta-analysis of chemotherapy on head and neck cancer. Lancet 2000; 355(9208):949-955

5 Okamoto I, Moriyama E, Fujii S, et al. Phase II study of carboplatin-paclitaxel combination chemotherapy in elderly patients with advanced non-small cell lung cancer. Jpn J Clin Oncol 2005;35(4):188-194

6 Sarkar A, Shahi U. Assessment of cancer care in Indian elderly cancer patients: a single center study. South Asian J Cancer 2013;2(4):202-208 
7 Hurria A, Togawa K, Mohile SG, et al. Predicting chemotherapy toxicity in older adults with cancer: a prospective multicenter study. J Clin Oncol 2011;29(25):3457-3465

8 Extermann M, Boler I, Reich RR, et al. Predicting the risk of chemotherapy toxicity in older patients: the Chemotherapy Risk Assessment Scale for High-Age Patients (CRASH) score. Cancer 2012;118(13):3377-3386

9 Soubeyran P, Fonck M, Blanc-Bisson C, et al. Predictors of early death risk in older patients treated with first-line chemotherapy for cancer. J Clin Oncol 2012;30(15):1829-1834

10 Extermann M, Bonetti M, Sledge GW, O’Dwyer PJ, Bonomi P, Benson AB III. MAX2-a convenient index to estimate the average per patient risk for chemotherapy toxicity; validation in ECOG trials. Eur J Cancer 2004;40(8):1193-1198

11 Globocan India 2018. Population fact sheets p. 1-2. Available from http://www.gco.iarc.fr/today/data/factsheets/populations/356-india-fact-sheets.pdf [accessed on May 11, 2020].

12 Parikh PM, Bakshi AV, Cancer in the elderly - Get ready for the epidemic. In: Gupta SB, editor. Medicine Update. Vol. 15. Mumbai, India: The Association of Physicians of India; 2005 827-831

13 Wildiers $\mathrm{H}$, Heeren P, Puts M, et al. International Society of Geriatric Oncology consensus on geriatric assessment in older patients with cancer. J Clin Oncol 2014;32(24):2595-2603

14 Hamaker ME, Schiphorst AH, ten Bokkel Huinink D, Schaar C, van Munster BC. The effect of a geriatric evaluation on treatment decisions for older cancer patients-a systematic review. Acta Oncol 2014;53(3):289-296

15 Freyer G, Geay JF, Touzet S, et al. Comprehensive geriatric assessment predicts tolerance to chemotherapy and survival in elderly patients with advanced ovarian carcinoma: a GINECO study. Ann Oncol 2005;16(11):1795-1800

16 Aaldriks AA, Maartense E, le Cessie S, et al. Predictive value of geriatric assessment for patients older than 70 years, treated with chemotherapy. Crit Rev Oncol Hematol 2011;79(2):205-212
17 Balducci L, Corcoran MB. Antineoplastic chemotherapy of the older cancer patient. Hematol Oncol Clin North Am 2000;14(1):193-212, x-xi x-xi.

18 Crawford J, Dale DC, Kuderer NM, et al. Risk and timing of neutropenic events in adult cancer patients receiving chemotherapy: the results of a prospective nationwide study of oncology practice. J Natl Compr Canc Netw 2008;6(2):109-118

19 Lyman GH, Morrison VA, Dale DC, Crawford J, Delgado DJ, Fridman M; OPPS Working Group. ANC Study Group. Risk of febrile neutropenia among patients with intermediate-grade non-Hodgkin's lymphoma receiving CHOP chemotherapy. Leuk Lymphoma 2003;44(12):2069-2076

20 Presant CA, Thompson E, Leberthon B, Vergara L, McGowen P. Effects of weekly paclitaxel or paclitaxel plus carboplatin on functionality and symptoms of geriatric patients with cancer as measured by a brief geriatric oncology module: a pilot experience. Cancer 2005;103(12):2623-2628

21 Puts MT, Monette J, Girre V, et al. Are frailty markers useful for predicting treatment toxicity and mortality in older newly diagnosed cancer patients? Results from a prospective pilot study. Crit Rev Oncol Hematol 2011;78(2):138-149

22 Bandeen-Roche K, Xue QL, Ferrucci L, et al. Phenotype of frailty: characterization in the women's health and aging studies. J Gerontol A Biol Sci Med Sci 2006;61(3):262-266

23 Reuben DB, Rubenstein LV, Hirsch SH, Hays RD. Value of functional status as a predictor of mortality: results of a prospective study. Am J Med 1992;93(6):663-669

24 Wolfson C, Wolfson DB, Asgharian M, et al. Clinical Progression of Dementia Study Group. A reevaluation of the duration of survival after the onset of dementia. N Engl J Med 2001;344(15):1111-1116

25 Hamer M, Bates CJ, Mishra GD. Depression, physical function, and risk of mortality: National Diet and Nutrition Survey in adults older than 65 years. Am J Geriatr Psychiatry 2011;19(1):72-78 